\title{
Transient lesion in the splenium of the corpus callosum due to rotavirus infection
}

\author{
Katarzyna Mazur-Melewska • Katarzyna Jonczyk-Potoczna • \\ Krystyna Szpura • Grzegorz Biegański • Anna Mania • Pawel Kemnitz • \\ Wojciech Służewski • Magdalena Figlerowicz
}

Received: 11 January 2015 / Accepted: 4 February 2015 / Published online: 17 February 2015

(C) The Author(s) 2015. This article is published with open access at Springerlink.com

\begin{abstract}
Transient signal changes in magnetic resonance imaging (MRI) of the splenium of the corpus callosum (SCC) can result from many different reasons, including encephalitis and encephalopathy caused by infection, seizures, metabolic disorders and asphyxia. We report a case of a 6-year-old Polish girl with rotavirus infection demonstrating a reversible SCC lesion on diffusion-weighted MRI images. She presented six episodes of generalized tonic seizures with mild acute gastroenteritis. Stool test for rotavirus antigen was positive. At the time of admission imaging showed the hyperintense region in T2-weighted and fluid-attenuated inversion-recovery MRI, a well-defined lesion in the splenium of the corpus callosum with restricted diffusion in diffusion-weighted MRI and no enhancement in post contrast T1-weighted imaging. Her first EEG showed slow brain activity in the posterior occipitotemporal portion, consisting mainly of theta waves with a frequency of $4.5-5.5 \mathrm{~Hz}$ and amplitude of $40 \mathrm{uV}$. The lesion had completely disappeared on follow-up MRI 10 days later. The patient recovered fully without any sequelae.
\end{abstract}

Keywords Rotavirus $\cdot$ Magnetic resonance $\cdot$ Corpus callosum $\cdot$ Child $\cdot$ Transient lesion

K. Mazur-Melewska $(\bowtie) \cdot$ K. Szpura $\cdot$ G. Biegański $\cdot$ A. Mania

P. Kemnitz $\cdot$ W. Służewski $\cdot$ M. Figlerowicz

Department of Infectious Diseases and Child Neurology, Karol

Marcinkowski University of Medical Sciences in Poznań, Szpitalna

Street 27/33, 60-578 Poznań, Poland

e-mail: katarzynam-m@wp.pl

K. Jonczyk-Potoczna

Department of Pediatric Radiology, Karol Marcinkowski University

of Medical Sciences Poznań, Szpitalna Street 27/33,

60-578 Poznań, Poland

\begin{abstract}
Abbreviations
ADC Apparent diffusion coefficient

CNS Central nervous system

DWI Diffusion-weighted MRI

EEG Electroencephalogram

FLAIR Fluid-attenuated inversion-recovery

MERS Mild encephalitis with a reversible splenial lesion

MRI Magnetic resonance imaging

SCC Splenium of the corpus callosum
\end{abstract}

Rotaviruses are responsible for significant gastrointestinal disease, primarily in children $<5$ years of age. Infection is localized in the intestines but some reports suggest any extraintestinal localization. Recently, a number of investigators have reported central nervous system (CNS) complications in association with rotavirus infections. Neurologic manifestations, ranging from benign convulsions to the viral encephalitis or encephalopathy, occur in approximately $2-5 \%$ of patients with rotavirus gastroenteritis [1-3]. A transient lesion in the splenium of the corpus callosum (SCC) associated with rotaviral infection has been reported in several cases, most of them in patients with encephalopathies. All of them were described in Asian countries like Japan or Korea [4, 5]. There have been no case reports to date of transient SCC lesions associated with rotaviral infections in Europe. We report the first case of a rotavirus gastroenteritis connected with mild encephalitis with a reversible splenial lesion (MERS) in Poland, Europe.

\section{Case report}

A 6-year-old girl was admitted to the hospital after three episodes of generalized, tonic seizures. Two days before admission, she developed watery diarrhoea and repeated vomiting. 
Her perinatal history was complicated by premature birth (34th week) with weight $2200 \mathrm{~g}$, Apgar scale -10. Her motor development was delayed in the first 2 years of life.

At admission, the girl complained about moderate abdominal pain and watery stools.

Physical examination showed a body temperature of $37.4{ }^{\circ} \mathrm{C}$, pulse rate of 110 beats $/ \mathrm{min}$ and blood pressure of $116 / 60 \mathrm{mmHg}$. The abdomen was soft, but painful. The bowel sounds were increased. Neurologic examination showed normal condition without pathological reflexes.

Laboratory findings presented the following: leukocyte count $4800 / \mu \mathrm{L}$, erythrocyte count $4,920,000 / \mu$, platelet count $253,000 / \mu \mathrm{L}$, haemoglobin $13.3 \mathrm{~g} / \mathrm{dL}$ and glucose $77 \mathrm{mg} / \mathrm{dL}$. The serum electrolyte levels were as follows: sodium $137 \mathrm{mmol} / \mathrm{L}$, potassium $4.89 \mathrm{mmol} / \mathrm{L}$, calcium $2.49 \mathrm{mmol} / \mathrm{L}$ and magnesium $19.3 \mathrm{mmol} / \mathrm{L}$. Aspartate aminotransferase was $49 \mathrm{U} / \mathrm{L}$ and alanine aminotransferase $26 \mathrm{U} / \mathrm{L}$. C-reactive protein was elevated to $2.57 \mathrm{mg} / \mathrm{dL}$. Serum samples showed an absence of antibodies against cytomegalovirus, EpsteinBarr, herpes simplex 1 and 2 viruses, Borrelia burgdorferi and Toxoplasma gondii. Lumbar puncture was not performed; cerebrospinal fluid could not be analysed for this patient.

Enzyme immunoassay detection of Rotavirus in the stool was positive. The stool culture and Adenovirus antigen were negative.

On the day of admission, the girl presented six episodes of generalized tonic seizures with lockjaw and flexion of the extremities. The first two episodes lasted up to $1 \mathrm{~min}$ and subsided after diazepam. The subsequent ones were shorter and disappeared spontaneously. Between these episodes, the patient's consciousness was disturbed. During further hospitalization, no seizures were observed.

Brain magnetic resonance imagination (MRI) was performed on the third day after admission using a 3-T scanner (MAGNETOM Spectra, Siemens Healthcare, Erlangen, Germany). T1-weighted examination was performed before and after i.v. administration of gadobutrol (Bayer Vital, Leverkusen, Germany, $0.1 \mathrm{mmol} / \mathrm{kg}$ body weight). The imaging showed the hyperintense region in T2-weighted and fluidattenuated inversion-recovery (FLAIR) MRI, a well-defined lesion in the splenium of the corpus callosum with restricted diffusion in diffusion-weighted MRI (DWI) and no enhancement in post contrast T1-weighted (Fig. 1).

An electroencephalogram EEG was performed with a 42channel DigiTrack system (Elmiko, PL), with electrodes placed in compliance with the international 20-electrode system. Her first EEG (the third day) showed slowed brain activity in the posterior occipitotemporal portion, consisting mainly of theta waves with a frequency of $4.5-5.5 \mathrm{~Hz}$ and amplitude of $40 \mathrm{uV}$.

She was treated with intravenous crystalloids without anticonvulsant control. After the MRI results, dexamethasone therapy $(0.4 \mathrm{mg} / \mathrm{kg} /$ day for 5 days) was started.
An MRI performed 12 days after admission revealed no abnormal signal in the splenium of the corpus callosum (Fig. 2). The EEG (awake and sleep) was completely normal.

The child was sent home without signs of neurological complications. At her follow-up examination 2.5 months later, she had age-appropriate motor skills and behaviour. Her EEG showed normal brain activity.

\section{Discussion}

The first observations about the possibility of CNS involvement with rotavirus infection were made by Salmi in 1978. It had been observed in children with rotavirus gastroenteritis with or without associated convulsions, encephalitis and cerebellitis and patients with Reye syndrome and with haemorrhagic brain shock $[1,3,5]$. Our patient manifested recurrent convulsions, confusion and disorientation without electrolyte disturbances. The EEG showed slowed brain activity, in contrast to Isik's description, who reported reversible sharp waves during rotavirus infection [6]. We confirmed the observation of Kobata et al., who described nine Japanese cases of MERS occurring during rotavirus infection. Among them, $89 \%(8 / 9)$ had encephalopathic patterns such as alterations in consciousness and abnormal behaviour. Transient SCC lesions have also been reported in encephalitis or encephalopathy induced by other viruses [4]. All reported patients with MERS had a mild clinical course and recovered completely without any sequelae. The mechanism behind these reversible lesions has been analysed by several authors. Tada et al. proposed that the lesions might be secondary to intramyelinic oedema due to disconnection of myelin layers, or else that an incursion of inflammatory cells and macromolecules combined with cytotoxic oedema causes decreased apparent diffusion coefficient levels. This might help to explain the decrease in diffusion levels in the splenium. Splenial lesions after seizures have also been reported in patients with epilepsy. Seizures impair glucose availability, conducted to reversible failure of cellular fluids regulation at the splenium. Reduced diffusion and normalization on follow-up imaging suggest transient intracellular oedema with diffusion restriction [7]. Axonal oedema caused by hyponatraemia or the development of an inflammatory infiltrate were suggested by Takanashi [8]. Oster et al. reported two bitemporal lobe epilepsy patients showing a transient SCC lesion. They suggested the possible mechanism involved a transient disturbance in energy metabolism and ionic transport resulting in reversible myelin vacuolization and intramyelin oedema due to excessive repetitive activity of the commissural projection from the temporal structure in these patients [9]. An analysis performed by Gröppel on 24 epilepsy cases with a focal lesion in the SCC did not present the relationship between the lesion and possible etiologic factors such as epilepsy or seizure types, 
Fig. 1 The hyperintense region in T2-weighted and fluidattenuated inversion-recovery (FLAIR) MRI, a well-defined lesion in the splenium of the corpus callosum with restricted diffusion in diffusion-weighted MRI (DWI) and no enhancement in post contrast T1-weighted (MRI Axial T1W precontrast (a) postcontrast (b) no lesion enhancement; Axial T2W (c), FLAIR (d), DWI (e) lesion marked using white arrow , ADC (f) - black arrow)

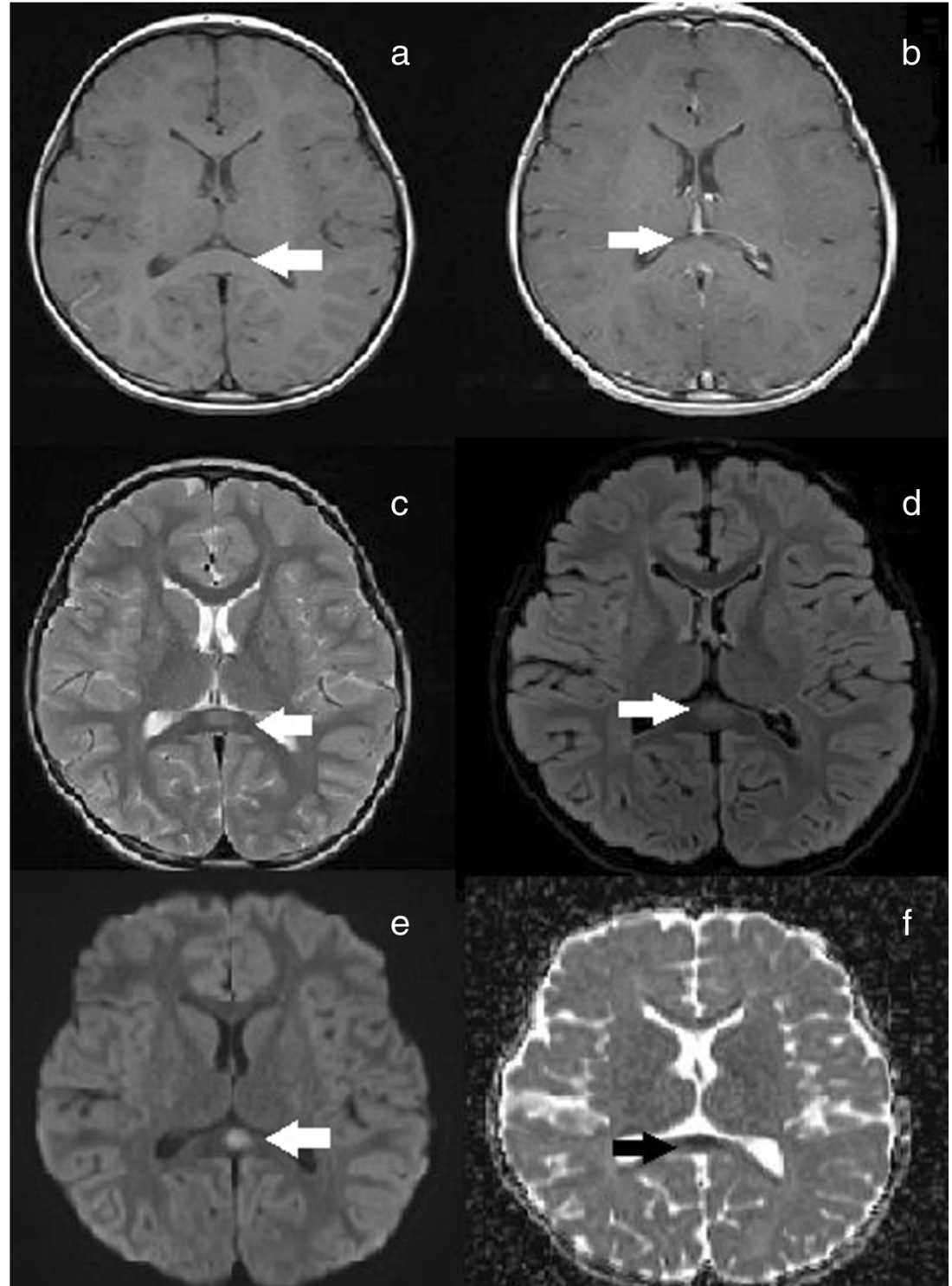

localization of interictal spikes and ictal EEG patterns or antiepileptic drugs [10]. A reversible SCC lesion could result from multiple conditions including rotaviral infection, gastroenteritis with electrolyte imbalance, seizures, encephalitis and encephalopathy. Taking this into consideration, our patient had many possible factors for the brain lesion's appearance.
Fig. 2 Magnetic resonance imaging $(M R I)$ obtained on the tenth day of illness shows complete disappearance of the splenial white matter lesions (MRI Axial T2W (a), FLAIR (b), DWI (c))

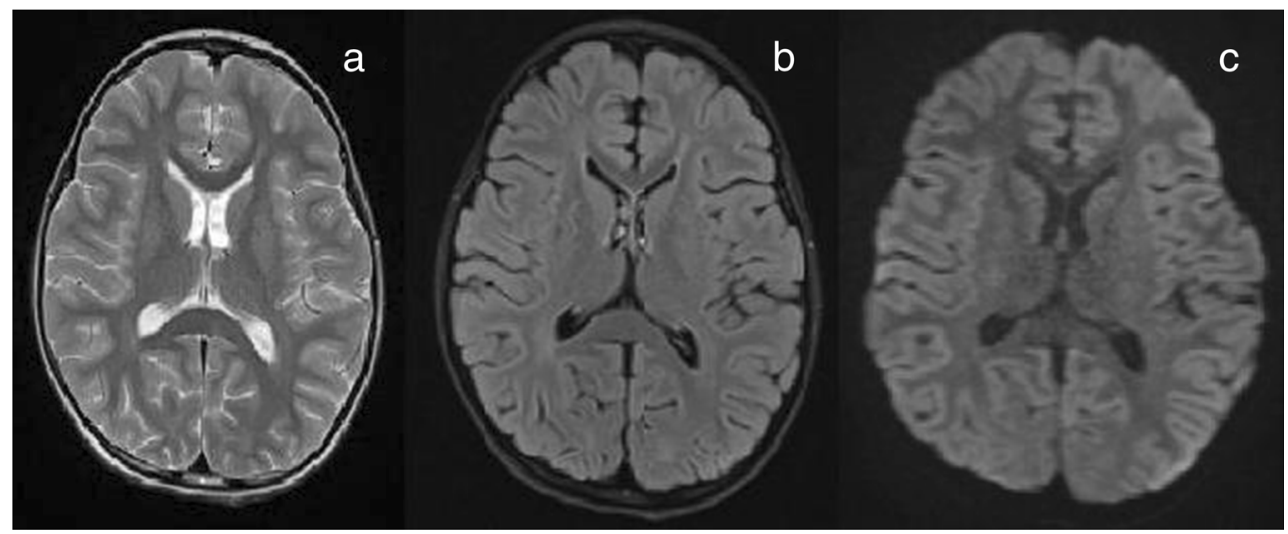


Open Access This article is distributed under the terms of the Creative Commons Attribution License which permits any use, distribution, and reproduction in any medium, provided the original author(s) and the source are credited.

\section{References}

1. Gröppel G, Gallmetzer P, Prayer D, Serles W, Baumgartner C (2009) Focal lesions in the splenium of the corpus callosum in patients with epilepsy. Epilepsia 50:1354-1360

2. Isik U, Caliskan M (2008) Reversible EEG changes during rotavirus gastroenteritis. Brain Dev 30:73-76

3. Jang YY, Lee KH (2010) Transient splenial lesion of the corpus callosum in a case of benign convulsion associated with rotaviral gastroenteritis. Kor J Pediatr 53:859-862

4. Kobata R, Tsukahara H, Nakai A, Tanizawa A, Ishimori Y, Kawamura Y, Ushijima H, Mayumi M (2002) Transient MR signal changes in the splenium of the corpus callosum in rotavirus encephalopathy: value of diffusion-weighted imaging. J Comput Assist Tomogr 26:825-828
5. Lynch M, Lee B, Azimi P, Gentsch J, Glaser C, Gilliam S, Chang H, Ward R, Glass R (2001) Rotavirus and central nervous system symptoms: cause or contaminant? Case reports and review. Clin Infect Dis 33:932-938

6. Modlińska-Cwalińska A, Mania A, Mazur-Melewska K, Kemnitz P, Januszkiewicz-Lewandowska D, Kuls K, Służewski W, Figlerowicz M (2012) Clinical and laboratory characteristics of rotavirus infections in children. Pediatr Pol 87:249-254

7. Oster J, Doherty C, Grant PE, Simon M, Cole AJ (2003) Diffusionweighted imaging abnormalities in the splenium after seizures. Epilepsia 44:852-854

8. Shiihara T, Watanabe M, Honma A, Kato M, Morita Y, Ichiyama T, Maruyama K (2007) Rotavirus associated encephalitis/ encephalopathy and concurrent cerebellitis: report of two cases. Brain Dev 29:670-673

9. Tada H, Takanashi J, Barkovich AJ, Oba H, Maeda M, Tsukahara H, Suzuki M, Yamamoto T, Shimono T, Ichiyama T, Taoka T, Sohma O, Yoshikawa H, Kohno Y (2004) Clinically mild encephalitis/ encephalopathy with a reversible splenial lesion. Neurology 63: 1854-1858

10. Takanashi J, Tada H, Maeda M, Suzuki M, Terada H, Barkovich AJ (2009) Encephalopathy with a reversible splenial lesion is associated with hyponatremia. Brain Dev 31:217-220 